\title{
Improved Low Voltage SEM Image Resolution Through the Use of Image Restoration Techniques
}

\author{
Matthew D. Zotta ${ }^{1}$, Yudhishthir P. Kandel ${ }^{1}$, Andrew N. Caferra ${ }^{1}$ and Eric Lifshin ${ }^{1}$ \\ ${ }^{1}$ College of Nanoscale Science and Engineering, SUNY Polytechnic Institute, Albany, NY, USA
}

Low voltage SEM or LV-SEM $(<5 \mathrm{keV})$ is particularly useful when observing insulators and beam sensitive materials, as well as for delineating fine surface structure [1]. Obtaining quality images at low beam energies can be challenging, however, because chromatic aberrations cause significant increases in the probe size. Since the probe size increases with the source energy spread, field emission guns (FEGs) or Schottky sources are preferred over thermionic sources. However, chromatic aberrations are still a problem because they lead to an increase in the probe size when the probe energy is decreased. To overcome this, manufacturers often use relatively high gun energies $(>5 \mathrm{keV})$ and introduce a retarding field at the end of the column to lower the landing energy of the electrons.

In this study, the beam retardation requirement is eliminated by working with a larger beam than the resolution needed and recovering the resolution by the method described by Lifshin et. al. [2] in which the point spread function (PSF) of the electron beam is determined and used to deconvolute a blurred image formed by a large probe. The PSF determination is based on a comparison between a reference image and an observed image acquired with the chosen operating conditions. In the previous work, the reference image was obtained with a high resolution microscope at a magnification approximating pixel level resolution. The blurred image was then obtained at the same beam energy and magnification, but with a larger probe size on a lower resolution microscope. In the current study, a single high resolution microscope is used where a reference image is obtained at high beam energy with pixel level resolution and used to establish the PSF for low beam energies. This requires knowledge of the materials in the reference standard and their respective secondary electron yields [3] so the image can be corrected for beam energy dependence. Furthermore, suitable adjustments for brightness and contrast were made. These corrections were used to calculate an approximate reference image at the lower beam energy and the subsequent PSF (Figure 3) from the low energy image. Figure 1 shows an Au-C Pella ${ }^{\circledR}$ image (c) calculated for $2 \mathrm{keV}$ from a reference image taken at $20 \mathrm{keV}$ using a TESCAN MIRA ${ }^{\circledR}$. The measured image at $2 \mathrm{keV}$ is given in (a) and the restored image in (b). The PSF obtained in this way was then applied to a Sn-C Pella ${ }^{\circledR}$ sample as shown in Figure 2 where (a) is the observed $2 \mathrm{keV}$ image, (b) is the restored image and (c) is a high resolution $20 \mathrm{keV}$ image taken of the same area for comparison of detail. The improvement in image resolution in the restored images can be estimated based on their contrast transfer functions (Figure 4) using the method developed by Joy [4].

\section{References:}

1. Michael, J.R., Scanning 33, 147-154 (2011).

2. Lifshin, E., Kandel, Y.P. and Moore, R.L., Microscopy and Microanalysis 20 (01), 78-89 (2014).

3. Lin, Y. and Joy, D. C., Surf. Interface Anal. 37, 895-900 (2005).

4. Joy, D.C., J Microsc 208 (Pt1), 24-34 (2002).

The authors acknowledge the support of Mr. Jeffrey Moskin, President of Nanojehm for providing the resources that made this study possible as well as TESCAN for providing instrumental support. 

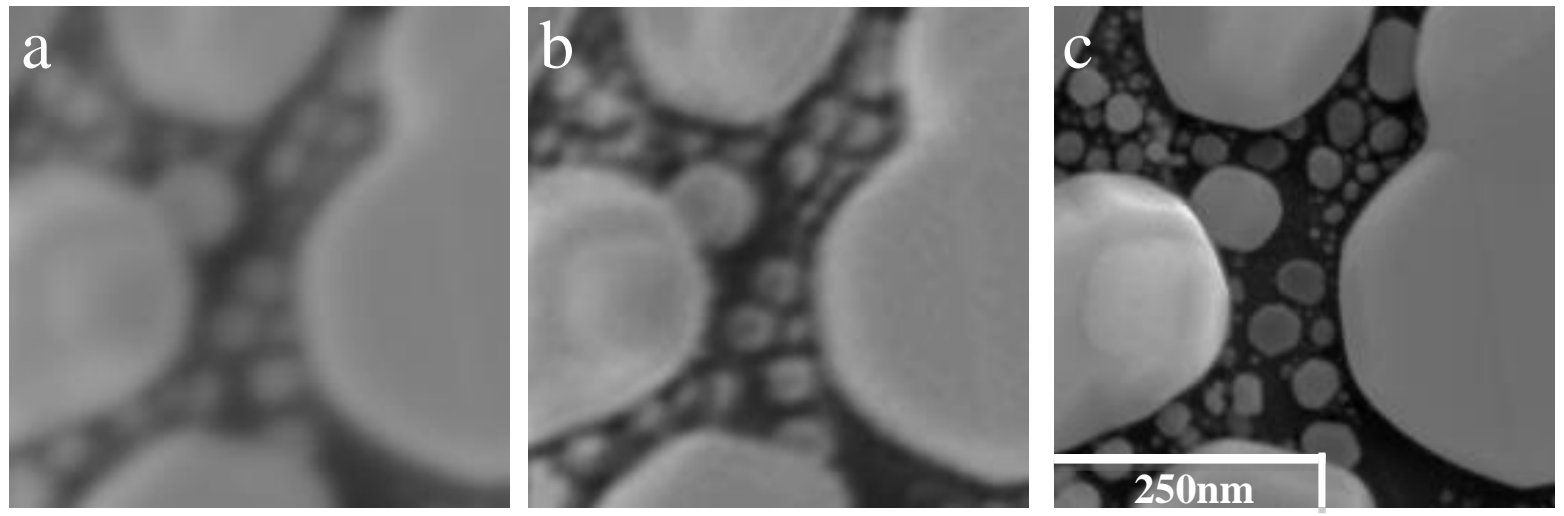

Figure 1. Au-C Pella ${ }^{\circledR}$ sample: (a) $2 \mathrm{keV}$ image, (b) restored $2 \mathrm{keV}$ image, (c) $20 \mathrm{keV}$ pixel level resolution image recalculated at $2 \mathrm{keV}$.
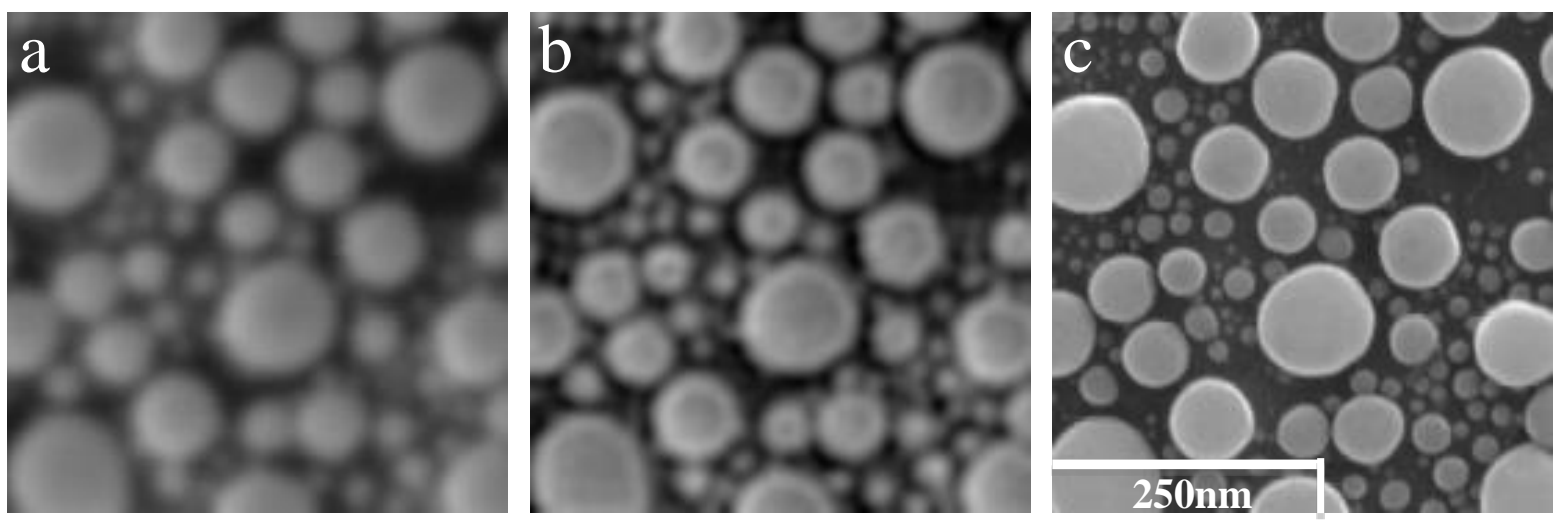

Figure 2. Sn-C Pella ${ }^{\circledR}$ sample: (a) $2 \mathrm{keV}$ image, (b) the restored $2 \mathrm{keV}$ image, (c) same area at $20 \mathrm{keV}$.

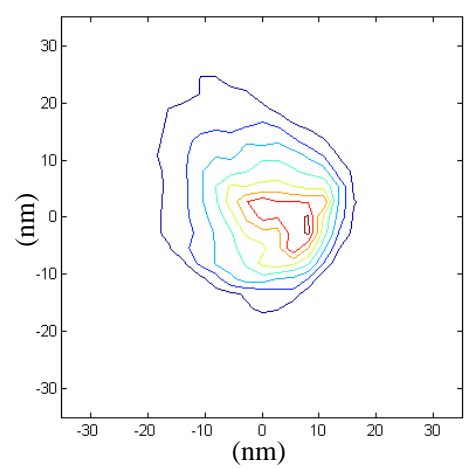

Figure 3. The PSF determined from the $2 \mathrm{keV}$ observed image relative to the $2 \mathrm{keV}$ reference image.
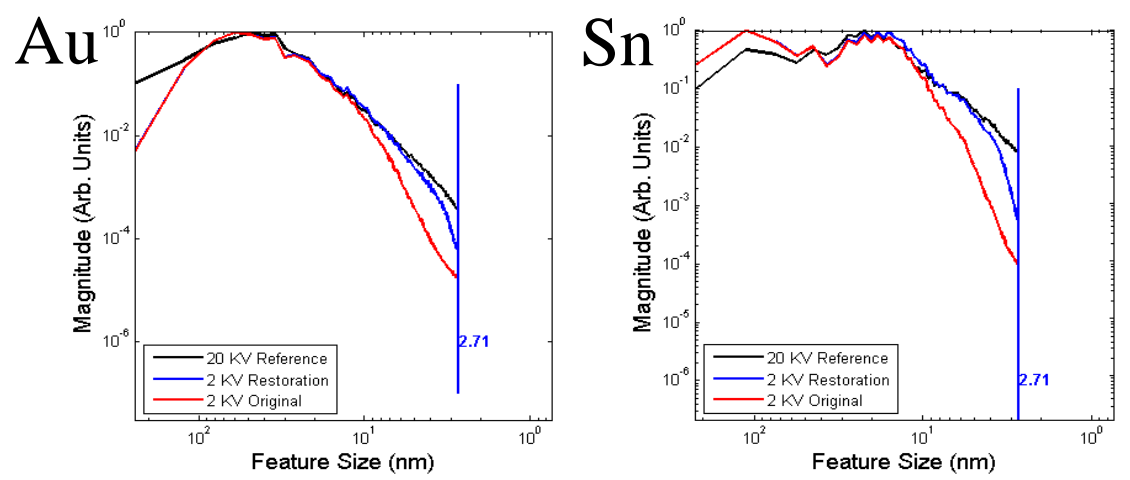

Figure 4. The contrast transfer functions for the $\mathrm{Au}$ and $\mathrm{Sn}$ $20 \mathrm{keV}$ and $2 \mathrm{keV}$ images and the $2 \mathrm{keV}$ restorations. 